\title{
An EHR with Teeth
}

Elsbeth Kalenderian'; John D. Halamka ${ }^{2,3}$; Heiko Spallek ${ }^{4}$

${ }^{1}$ Harvard Dental Center, Harvard School of Dental Medicine, Boston, MA;

${ }^{2}$ Beth Israel Deaconess Medical Center, Harvard Medical School;

${ }^{3}$ Beth Israel Deaconess Medical Center, Roxbury Crossing MA;

${ }^{4}$ Faculty of Dentistry, University of Sydney, Australia

\section{Correspondence to:}

Elsbeth Kalenderian, DDS, MPH, PhD

Chair, Oral Health Policy and Epidemiology

Chief of Quality, Harvard Dental Center

Harvard School of Dental Medicine

188 Longwood Avenue, REB 206

Boston, MA 02115

Telephone: 617-432-1455

Mobile: 617-947-7645
Appl Clin Inform 2016; 7: 425-429

http://dx.doi.org/10.4338/ACl-2015-09-LE-0124

received: October 9, 2015

accepted: March 6, 2016

published: May 25, 2016

Citation: Kalenderian E, Halamka JD, Spallek H. An

EHR with teeth. Appl Clin Inform 2016; 7: 425-429

http://dx.doi.org/10.4338/ACl-2015-09-LE-0124 
During a routine visit, Dr. Vera Cruz, a pediatrician, notes that a 3-year-old boy has a tooth of darker shade with a pus-emitting abscess next to it. The child's mother confirms that her son has been eating and sleeping poorly. Dr. Cruz tells the mother to seek out dental care urgently. Although she attempts to follow up with the family a few days later, Dr. Cruz is unable to confirm that the child has received treatment until the family returns for a well-child visit one year later.

Mr. Smith is an established patient in Dr. Nguyen's dental practice. Prior to completing several fillings, Dr. Nguyen notes that, as at the previous visit, Mr. Smith's blood pressure is elevated and measures 165/105. Dr. Nguyen advises Mr. Smith to discuss his high blood pressure findings with his physician, but cannot confirm that Mr. Smith has sought evaluation.

Mr. Jones takes Coumadin as part of his management regime for atrial fibrillation. He presents in severe pain to a community health center dental clinic and is diagnosed with numerous badly decayed and broken off teeth, advanced gum disease and a dental abscess. Mr. Jones prefers to have all five involved teeth extracted. Dr. Nuñez needs to confirm that his INR is within a safe range to perform the surgery. The health center's receptionist spends 45 minutes trying to reach Mr. Jones' physician. By the time she obtains the relevant information, $\mathrm{Mr}$. Jones feels weak, dizzy, and is sweaty as his blood sugar is too low from not having eaten prior to his dental appointment. Dr. Nuñez feels uncomfortable performing the surgery given the low blood sugar symptoms but rather performs a quick I\&D, provides prescriptions for pain and antibiotic medications, and an appointment to come back next week.

In all of these examples, optimal patient care was thwarted by the inability of medical and oral healthcare providers to share crucial health information about a particular patient in their care. This letter is a call to all stakeholders of contemporary EHRs, (1) vendors producing them, (2) providers using them, (3) payers receiving data from them, and (4) patients benefiting from effective and efficient information access, to implement existing industry standards towards true interoperability between dental and medical EHRs overcoming all real and perceived barriers. While there is no clear definition of interoperability, it can be described as "a system, whose interfaces are completely understood, to work with other products or systems, present or future, without any restricted access or implementation" [1]. More importantly, it needs to include a syntactic level, i.e. interoperability of data formats and communication protocols, as well as a semantic level, i.e. meaningful and accurate interpretation of the information exchanged. Dentists perform irreversible surgical procedures, prescribe and administer medications including antibiotics and agents for sedation and anesthesia, and screen for illnesses including oral cancer, diabetes, and hypertension-they are part of the interprofessional health care team as defined in the Core Competencies for Interprofessional Collaborative Practice [2]. Many oral-systemic health connections are known today, such as the association of periodontitis with cardiovascular disease as well as acute and stable coronary artery disease [3] and many more are discussed and confirmed [4-6] emphasizing the importance of just-in-time two-way communication of detailed health information between the dental and medical team. This request for interoperability has been confirmed by physicians indicating that their medical decisions would be impacted by access to patients' oral health record [7], but has rarely been implemented. Dentists and physicians can, for instance, make use of seamless data exchange when caring for patients with diabetes mellitus by embracing a medical-dental collaboration when identifying undiagnosed diabetes in the dental care setting or when identifying undiagnosed periodontal disease in primary care settings [8].

There are many technical hurdles, such as the often incompatible implementations of the Continuity of Care Document (CCD) standard by different EHR vendors, that must be overcome for information exchange using the Health Information Exchange (HIE) route between medical and dental EHRs. We find unnecessary regulatory complexity, such as the confusing requirements in Meaningful Use, the HIPAA Omnibus Rule, the Affordable Care Act, ICD-10 and the Medicare Access \& CHIP Reauthorization Act of 2015 (MACRA) that have been developed without input from dental providers or with the specifics of the dental care environment in mind. There is a lack of business imperative, as dental practitioners in solo practice have few or no reasons to embrace change and innovation that comes along with interoperability. We miss an enabling infrastructure to accelerate interoperability, such as a national provider directory, a master patient index/relationship locator service, a consent service, a certificate management service, and test beds for developers to exercise these services. In order to address these obstacles, we need a partnership of government, payers, pro- 
viders, patients, and health IT developers working together to achieve common goals. Stakeholders need to acknowledge the health benefits that can be gained from supporting the information exchange that helps us understand the oral-systemic health connection. If we want to support interprofessional collaborative practice, we need to be able to use FHIR to read a medical and dental provider directory (could be hosted by government such as CMS as part of the national provider identifier or the private sector such as Surescripts, DirectTrust, or an HIE) and send a Direct message to that provider for referrals [9].

Consensus has emerged that an "organized and multifaceted infrastructure needs to be in place for the efficient and effective implementation of the oral health competencies ... [that] enable[s] systems to optimize care coordination across medical and dental care environments to improve patient outcomes." Such an approach acknowledges that "[h] ealth care systems are complex operational structures that include access to care, provision of clinical services, and the underlying foundation that supports implementation of the oral health core clinical competencies" as recently outlined by the 2014 U.S. Department of Health and Human Services Health Resources and Services Administration's report "Integration of Oral Health and Primary Care Practice" [10]. The same report outlines four main recommendations that go beyond the technical details: (1) apply oral health core clinical competencies within primary care practices, (2) develop an infrastructure that is interoperable, accessible across clinical settings, and enhances adoption of the oral health core clinical competencies, (3) modify payment policies to efficiently address costs of implementing oral health competencies and provide incentives to health care systems and practitioners, and (4) execute programs to develop and evaluate implementation strategies of the oral health core clinical competencies into primary care practice.

Phase 2 of the Meaningful Use Incentives Program of the HITECH Act mandates that at least $80 \%$ of patients seen by eligible providers have height, weight, blood pressure, and smoking status recorded. At the moment, the program does not differentiate among various healthcare professionals, thus dentists [11] are participating in these screening programs performing basic risk assessments and prevention measures. However, the collected information may never reach the rest of the patient's healthcare team -in other words, the valuable blood pressure measurements are locked up in the dental EHR.

There are promising examples of regional data exchanges, such as in Massachusetts where 160 different EHRs in 400 organizations exchange two million detailed clinical summaries every month. Or, large-scale government contracts that require the integration of medical and dental record system, such as the 2015 US Department of Defense electronic health record system contract that will include service personnel dental records [12] that acknowledges the importance of the oral-systemic connection for health outcomes. In addition, there are stakeholder-driven approaches that result in increased interoperability, such as the Argonaut Project (http://www.argonautproject.org) that promotes an Internet standards-based architecture that enables expanded information sharing: every major EHR vendor agreed to create application programming interfaces (APIs) that enable 3rdparty modules to be added to existing EHRs. However, the same cannot be said about most dental EHRs, which are not interoperable with medical EMRs, thus rendering these dental EHR databases into data silos. Additionally, for interoperability to be meaningful, the shared information needs to be in readable and accessible format. I.e. while a dentist needs a detailed tooth chart when charting oral health examination findings, a physician may wish to see a more succinct oral health summary. How do we convert these data silos into sources of knowledge and improve patient outcomes based on that knowledge?

The focus to date on interoperability has concentrated on improving standards, which is necessary but not sufficient. There are other enablers that are pre-requisites to universal sharing of healthcare data. These include:

a. A voluntary patient identifier. In the US there is no simple means to link patient identities across care providers. Demographic matching of name, gender, and date of birth is not precise enough. The US is unlikely to mandate a national identifier given the existing Clinton era (1999) executive order prohibiting HHS funding for discussion of,

b. A national provider directory that enables clinicians to easily route clinical summaries to each other. 
c. Alignment of incentives and payment reform that reimburses clinicians for outcomes and quality rather than fee for service. Interoperability across disciplines and among providers and integration of workflow becomes an economic imperative.

d. Customer pressure on vendors to support their interoperability needs. Although many medical EHR vendors already recognize they can no longer sell an EHR lacking interoperability features, dental EHRs have yet to feel that market threat.

e. Enabling patients to be the stewards of their own data. By providing access to all records - medical and dental - to patients, they can facilitate sharing of information among all their providers.

Dental and medical schools and residency training programs are embracing interprofessional education and guided by their accrediting bodies are preparing all health science students for interprofessional practice [2]. Academic health professional leaders are spearheading the interprofessional health system, recently coined a Continuous Learning Health System [13]. Any healthcare system that claims to provide comprehensive healthcare-which undoubtedly includes oral health-must ensure that all providers are able to access and edit only one health record for each patient regardless of physical storage location and ownership. Medical and dental clinicians need to be able to seamlessly collaborate using all existing health information about their patients regardless of origin. For all of this to happen we suggest that (1) tools for exchanging health information need to be simplified; and that (2) the diverse piecemeal of state privacy laws impede information exchange and need to be streamlined. We expand Halamka's earlier suggestion that a "crisp set of requirements for care management and care coordination with defined metrics of success" will also include measures of oral health integration with general health partners.

Information technology can now be used to bring information services to distant locations, dentists send digital impressions across the globe to dental laboratories to manufacture fixed restorations, physicians have their radiographs interpreted at night on the other side of the globe where it is daytime, but a dentist and physician within the same building remain unable to share information about their mutual patients. As the healthcare technology field further develops, we strongly urge all stakeholders, EHR vendors, providers, patients and payers, to insist on the inclusion of the oral health component into an EHR. President and CEO of the American Dental Education Association, Dr. Richard Valachovic, asked the obvious question: How did the oral cavity get separated from the rest of the body?

\section{Human Subjects Protections}

No human subjects were involved in this project.

\section{Conflict of Interest}

The authors declare that they have no conflicts of interest in this project.

\section{Acknowledgement}

The authors would like to express their gratitude to Drs. Lisa Simon and Romesh Nalliah who triggered the thought process that ultimately generated this paper. They also inspired the patient vignettes that introduce the topic to the reader. The authors' gratitude is also directed to Michael Dziabiak, MLIS, Media Specialist at the School of Dental Medicine, University of Pittsburgh, for preparing the bibliography and formatting the manuscript for submission. In addition, the manuscript greatly benefitted from the thoughtful comments of the anonymous reviewers. 


\section{References}

1. Definition of interoperability. Interoperability Working Group [cited 2016 Feb 18]. Available from: http://interoperability-definition.info/en/.

2. Schmitt M, Blue A, Aschenbrener CA, Viggiano TR. Core competencies for interprofessional collaborative practice: reforming health care by transforming health professionals' education. Acad Med 2011; 86(11): 1351.

3. Hyvarinen K, Mantyla P, Buhlin K, Paju S, Nieminen MS, Sinisalo J, Pussinen PJ. A common periodontal pathogen has an adverse association with both acute and stable coronary artery disease. Atherosclerosis 2012; $223(2): 478-484$.

4. Glick M. The oral-systemic health connection: a guide to patient care. Chicago: Quintessence Publishing; 2014.

5. Lamster IB. Diabetes mellitus and oral health: an interprofessional approach. Ames, IA: Wiley-Blackwell; 2014.

6. Genco RJ, Williams RC. Periodontal disease and overall health: a clinician's guide. Yardley, PA: Professional Audience Communications, Inc.; 2010.

7. Acharya A, Mahnke A, Chyou PH, Rottscheit C, Starren JB. Medical providers' dental information needs: a baseline survey. Stud Health Technol Inform 2011; 169: 387-391.

8. Wisconsin diabetes mellitus essential care guidelines. Madions, WI: Wisconsin Diabetes Prevention and Control Program; 2012 [cited 2016 Feb 18]. Available at: https://www.dhs.wisconsin.gov/publications/ p4/p49356.pdf.

9. Halamka JD. The path forward for meaningful use. 2015 Nov 11 [cited 2015 Dec 14]. In: Life as a Healthcare CIO. Boston, MA. Available from: http://geekdoctor.blogspot.com/2015/11/the-path-forward-formeaningful-use.html.

10.Integration of oral health and primary care practice. U.S. Department of Health and Human Services Health Resources and Services Administration. 2014 Mar 31 [cited 2015 Dec 14]. Available from: http://www.hrsa.gov/publichealth/clinical/oralhealth/primarycare/integrationoforalhealth.pdf

11. Kalenderian E, Walji M, Ramoni RB. „Meaningful use“ of EHR in dental school clinics: how to benefit from the U.S. HITECH Act's financial and quality improvement incentives. J Dent Educ 2013; 77(4): 401-415.

12.Department of Defense awards Leidos \$4.3 billion contract. PR Newswire. 2015 Aug 19 [cited 2015 Dec 14]. Available from: http://www.prnewswire.com/news-releases/department-of-defense-awardsleidos-43-billion-contract-300130856.html

13. Friedman C, Rubin J, Brown J, Buntin M, Corn M, Etheredge L, Gunter C, Musen M, Platt R, Stead W, Sullivan K, Van Houweling D. Toward a science of learning systems: a research agenda for the high-functioning Learning Health System. J Am Med Inform Assoc 2015; 22(1): 43-50. 